\title{
Construction of Community Sports Medicine Health Service System Dependent on Gait Classification Algorithm
}

\author{
W. CHUN AND Z. BING \\ School of Continuing Education, Chengdu Sport Institute, Chengdu, China, 610041, Department of Physical Education, Tang- \\ shan Normal University, Tangshan, China, 063000
}

Chun and Bing: Community Sports Medicine Health Service System Dependent on Gait Classification

\begin{abstract}
As the cornerstone of health service, the state of the community sports medicine cannot be ignored. Based on the gait classification algorithm, a kind of community sports medicine health service system is established. The gait classification algorithm is applied to carry out empirical analysis. In addition, time series is added to make vertical comparison of the health states of the community sports medicine health system in China in the recent years. The empirical results showed that the overall health level of the community sports medicine in China is relatively low and there is a lot of opportunities for improvement. Finally, countermeasures and suggestions are put forward from the aspects of improving the medical innovation ability, increasing the input in the technology acquisition and transformation, constructing an open and good community sports medicine health service system and so on.
\end{abstract}

Key words: Community sports medicine, health service system, healthiness, gait classification algorithm

At present, the community sports medicine in China is in the stage of superposition of three phases. In this stage, the structural contradictions are prominent and there is no room for any delay of the industrial transformation and upgrading ${ }^{[1,2]}$. With the rise of the innovation driven development as a national strategy, innovation and entrepreneurship is in the ascendant. The strategic position of the community sports medicine has become increasingly apparent. In addition, it has played a key role in promoting the traditional industries to improve quality and efficiency and leading the development of the emerging industries ${ }^{[3,4]}$. In the new community sports medicine era characterized by the knowledge community sports medicine, the medical improvement of information is reshaping the industrial organizations. Industrial innovation has become an important starting point for the transformation of the community sports medicine. In addition, the close integration between the innovation subjects has made the innovation activities more efficient ${ }^{[5,6]}$. Under the new normal of the community sports medicine, community sports medicine, as a strategic and leading industry that is knowledge intensive and technology intensive, has become an important force to drive the development of the community sports medicine and the society in our country. However, it also faces important adjustments ${ }^{[7,8]}$. The problems of low technology conversion rate, weak intellectual property protection, lack of forwardlooking innovation policies and so on have restricted the healthy development of the community sports medicine in our country to a great extent ${ }^{[9]}$. Facing the vigorous development of the community sports medicine in the world and the tremendous challenges brought about by the increasingly fierce international competition, the community sports medicine in our country must realize the transformation from the single subject innovation to the chain innovation and community sports medicine health system, so as to stimulate the innovation vitality and improve the construction efficiency ${ }^{[10]}$. In this paper, the health mechanism of the community sports medicine is explored, which has clarified the internal operation principle of the system. In addition, the community sports medicine health system in our country is taken as an example to carry out empirical analysis, and then the theoretical basis and the countermeasures for the sustainable development of the community sports medicine health are provided accordingly ${ }^{[11,12]}$. The health research of the health system originated 
from abroad, and the studies on the healthiness were mainly focused on the concept connotation and the performance characteristics ${ }^{[13]}$. The health system is regarded as a body and it is considered that whether it is healthy or not is dependent on two aspects as the following, with resilience and maintaining the internal stabilization; health is defined from three dimensions, that is, vitality, organizational structure and resilience; for the definition of the healthy system, if a system is stable, sustainable and energetic and it can maintain its own condition over time and have resistance to external pressure, then the system is healthy ${ }^{[14,15]}$. On the basis of the studies published abroad, researchers have shifted their research focus to the health evaluation. Health service system is constructed from the 4 dimensions of biology, social community sports medicine, human health and social public policy. It is put forward that not only the whole should be analyzed when the evaluation is carried out, but the internal components of the system should be analyzed separately. Based on two aspects, that is, the classification of the health system and the factors that affect the health, the evaluation is carried out. In addition, the model of the health service system is established by drawing on the engineering fuzzy set theory. The external interference and stress are the main factors that lead to the changes in the health condition and the concept of health level is put forward that nested hierarchical integration can be carried out from two dimensions of the time and space to measure the health level.

Since then, some investigators innovated the health evaluation methods and further deepened the health evaluation theory. It is put forward that the evaluation indicator system can be constructed from two aspects, that is, the internal indicators and the external indicators of the health system. It is considered that the reason why the effectiveness of the health service system is insufficient is the failure to determine the evaluation criteria and the frame of reference in an effective manner, as well as the failure to distinguish between the human stress and the natural disturbance correctly; and the 5 basic principles of health can be described as the following: dynamic, hierarchical, creative, relevant and fragile in accumulation, thus the specific evaluation method of the VOR model (vigor - organizational structure - recovery) is expounded accordingly. Some other researchers have extended the theory of health through the application of the new concepts and new ideas in the community sports medicine, explored its connotation and established an econometric model and initially constructed a theoretical framework of the health carrying capacity based on the health and established a framework of urban vitality indicator accordingly.

\section{PRINCIPLES OF THE COMMUNITY SPORTS MEDICINE HEALTH}

Survival is the first demand of the community sports medicine health system and development is the inherent requirement of the community sports medicine health system. The development of the community sports medicine health system can be divided into 2 stages as the following, the incubation stage and the diffusion stage and the operation mechanisms in the stages are referred to as the stabilization mechanism and the expansion mechanism, respectively. These 2 mechanisms are coupled with each other in the operation at the same time, interact and correlate with each other to jointly promote the rapid flow of the innovation resources and the quick gathering of the innovation factors, thereby enhancing the system innovation capacity and improving the construction efficiency of the system. The external manifestation of the stabilization mechanism is sustainability, which dominates the incubation stage and is embodied in the self-survival instinct of the system; the external manifestation of the expansion mechanism is the competitiveness, which dominates the diffusion stage and is reflected in the self-expansion tendency of the system. The mechanism of stabilization and expansion of the community sports medicine health system is shown in fig. 1.

\section{Stabilization mechanism, sustainability:}

The incubation stage is the initial stage of the community sports medicine health system, which has directly reflected the primary situation of the system

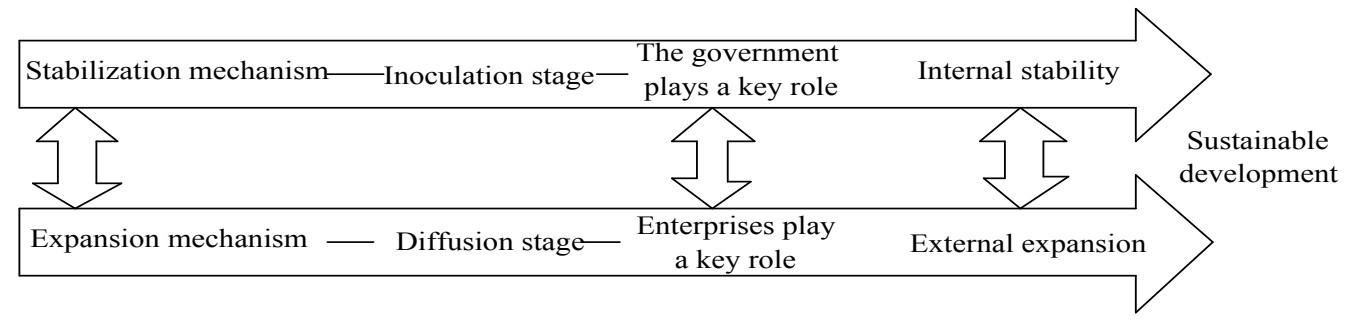

Fig. 1: Community sports medicine health mechanism 
and can be regarded as the survival risk period of the system. When the community sports medicine health system is in the incubation stage, in case it encounters a strong external impact or a major change in the internal structure, it is likely to cause the system to crash. When the community sports medicine health system has just been formed, the innovation chain within the system is not perfect and sturdy yet. Once the external community sports medicine health service system changes drastically or the system splits internally, it will lead to the deviation of the innovation health from the equilibrium or even falling into the crash trap of the system. A community health medicine health system with relatively strong vitality will definitely have a relatively stable structure. The number of the innovation subjects, the innovation output capacity and the construction efficiency in the system all present a relatively stable state. Each innovation subject and the community sports medicine health service system have coordinated operation and steady development. In this process, the government will play a key role. As a source of the policy innovation, the government should pay attention to the protection of the naive community sports medicine in the equation of the community sports medicine planning, the introduction of the medical policies, the cultivation and introduction of the talents, the encouragement of the scientific research activities, the regulation of the community sports medicine health service system and other fields, so as to safeguard the healthy and stable operation of the system. Therefore, the incubation stage shows whether the community sports medicine health system can continue to exist as well as the internal stabilization is related to the survival of the innovation subjects in the innovation chain.

\section{Expansion mechanism, competitiveness:}

The diffusion stage is the development stage of the community sports medicine health system, which has directly reflected the strength of the competitiveness of the system and can be regarded as the system quality change period. The community sports medicine health system has a natural tendency of self-expansion. After the self-stabilization in the incubation stage, the system itself has established certain barriers in the innovation chain and the knowledge chain and has the foundation and ability for the external expansion. A community sports medicine health system with strong competitiveness shows a kind of strong gravitational field with high construction efficiency, fast knowledge flow rate, short innovation cycle, strong community sports medicine and strong innovation barriers. The internal operation speed of the entire community sports medical health system is speeded up, which can have a strong impact on the external community sports medical health service system. To a certain extent, it will attract the other community sports medicine health systems and absorb the other community sports medicine health systems into its own system, making them a certain link in the innovation chain within the system. Throughout the entire process, the role of the community sports medicine is extremely important. The community sports medicine, as a source of the innovative applications, should develop new products with the market demand as the guidance, carry out research on the new technologies, focus on the user experience, flexibly adjust the research and development activities, and use its strong comprehensive innovation capacity to form its own core competitiveness in the market, technology, brand and other aspects, so as to create its own moat. The community sports medicine health system in the diffusion stage presents the characteristics as the following, the acceleration of the basic theoretical research, the increase of the knowledge flow frequency, a strong government guidance policy, enhancement of the technology research and development capabilities, shortening of the product promotion cycle, preemption of the frontier areas with various innovations, expansion of market share, continuous expansion of the innovation space and the enhancement of the synergistic advantage, thereby building the momentum for further development in the future.

\section{Gait classification algorithm:}

A clear understanding of the inherent essence of the community sports medicine health should be carried out, that is, the factors that may affect the community sports medicine health service system should be mapped into the discriminant space in an effective manner. If there are $m(m>3)$ factors present that may affect the normal running of the community sports medicine health, it is required is functioning normally, it is required that the community sports medicine health can reach $n(n>1)$ at this point. In addition, both of these are linked through the m-dimensional to $\mathrm{n}$-dimensional mapping. If $\mathrm{m}$ stands for the input number of the gait classification algorithm, $n$ stands for the output number of the gait classification algorithm, then for the m-dimensional Euclidean space $R^{[+]}$, there is a bounded subset $\mathrm{A}$ in the m-dimensional Euclidean space. In addition, there is a mapping present, so that it can correspond to a bounded subset $\mathrm{F}$ (A) in the n-dimensional Euclidean spacer $R^{H}$. Hence, the mapping relationship can be 
expressed as the following $F: A \& R^{\exists+\%} \rightarrow R^{H}, Y=F(X)$ $\mathrm{A}=(\mathrm{X}, \mathrm{Y})$ stands for the training set. After learning, the following mapping $\mathrm{G}$ can be obtained, which meets the

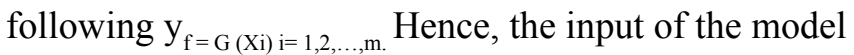
at this point is set as the evaluation indicator system of the community sports medicine health model, that is, $X=\left(x_{1}, x_{2}, \ldots X_{[+]}\right.$. The early warning result is used as the output of the method, that is, $Y=\left(y_{1}, y_{2}, \ldots Y_{H}\right)$, and the model is tamed with the characteristic degree of the typical service system, so as to ensure that the input vectors are different, and that the output values thus obtained are different as well. In this way, the input and output are linked by the mapping. In addition, the mapping is a highly nonlinear mapping, which is established based on the simple nonlinear algorithm and can express the problem of community sports medicine health in an effective manner, that is, the gait classification algorithm has been realized.

The health problems of the community sports medicine are very complicated and are nonlinear. Therefore, it is possible to obtain the amount of the gait classification problem by means of conversion. Hence, the optimal classification plane can be obtained in the gait classification. The slack variable $\xi_{r}\left(\xi_{r} \geq 0, i=1,2, \cdots, n\right)$ is introduced. In addition, it is ensured that the plane can make the hyperplane $\mathrm{W}^{\mathrm{T}} \mathrm{x}+\mathrm{b}=0$ reach the key culture as the following $\mathrm{y}=\left(\mathrm{w}^{\mathrm{T}} \mathrm{x}_{\mathrm{r}}+\mathrm{b}\right) \geq 1-\xi_{\mathrm{r},}$ If $0\left\langle\xi_{\mathrm{r}}\langle 1\right.$ is met, it indicates that correct classification of the sample point $X_{r}$ can still be achieved; while if $\xi_{r} \geq 1$ is met, the sample point $X_{r}$ will be misclassified at this point. Hence, the gait classification algorithm is introduced as the following $\Psi(\mathrm{w}, \xi)=1 / 2 \mathrm{w}^{\mathrm{T}} \mathrm{W}+\mathrm{C} \sum_{\mathrm{r}=1}{ }^{\mathrm{H}} \xi_{\mathrm{r},} \mathrm{C}$ is a normal number, that is, a penalty factor. Hence, the gait classification problem can be achieved through the second level planning problem as the following,

$$
\begin{aligned}
& \operatorname{Max} \sum_{r=1}{ }^{{ }^{H}} a_{r}-1 / 2 \sum_{r=1}{ }^{H} \sum_{f=1}{ }_{r} a_{r} a_{f} y_{r} y_{f}\left(x_{r}^{T} x_{f}\right) \\
& \text { s.t } 0 \leq a_{r} \leq C, i=1, \cdots, n \\
& \sum_{r=1}{ }^{{ }^{H}} a_{r} y_{r}=0
\end{aligned}
$$

The aforementioned transformation is relatively complicated and in general situation, is not easy to implement. However, if it is in a high-dimensional space, it is only required to use the inner product operation, then the above transformation can be achieved. In addition, even the knowledge of its specific transformation form is not required in accordance with the relevant functional theory. If the kernel algorithm $\mathrm{K}\left(\mathrm{X}_{\mathrm{r}}, \mathrm{X}_{\mathrm{f}}\right)$ is in line with the Mercer condition, it must have a corresponding relationship with the inner product of the gait classification. Therefore, if it is in the sufficient classification plane, through the appropriate inner product algorithm $\mathrm{K}\left(\mathrm{X}_{\mathrm{r}}, \mathrm{X}_{\mathrm{f}}\right)$ of the nonlinear transformation, the linear classification can be obtained. In addition, the computational complexity will not be increased in this way. In the WLR method, the gait classification algorithm can be converted to obtain different gait classification algorithms. The specific form of the gait classification is shown as the following, $\mathrm{K}\left(\mathrm{x}, \mathrm{x}_{\mathrm{r}}\right)=\exp \left\{-\left\|\mathrm{x}-\mathrm{x}_{\mathrm{r}} / 2 \sigma^{2}\right\|^{2}\right\}$. Compared to the conventional methods, the main difference of the algorithms thus obtained is that each algorithm has a corresponding support vector and the input weight can be determined in accordance with the algorithm. Then, the final gait classification can be expressed as the following $\mathrm{Q}(\mathrm{a})=\sum_{\mathrm{r}=1}^{\mathrm{r}} \mathrm{a}_{\mathrm{r}}-1 / 2 \sum_{\mathrm{rf}=1}^{\mathrm{r}} \mathrm{a}_{\mathrm{r}} \mathrm{a}_{\mathrm{f}} \mathrm{y}_{\mathrm{r}} \mathrm{y}_{\mathrm{f}} \mathrm{K}\left(\mathrm{x}_{\mathrm{r}} \cdot \mathrm{x}\right)$. $\mathrm{T} \mathrm{h}$ e corresponding gait classification can be obtained as the following $\mathrm{f}(\mathrm{x})=\operatorname{sgn}\left\{\sum_{\mathrm{r}=1}^{\mathrm{k}} \mathrm{a}_{\mathrm{r}}^{*} \mathrm{y}_{\mathrm{r}} \mathrm{K}(\mathrm{x}, \mathrm{x})+\mathrm{b}^{*}\right\}$, $\mathrm{x}$ stands for the support vector, $\mathrm{x}_{\mathrm{r}}$ stands for the unknown vector, $\mathrm{a}_{\mathrm{r}}^{*}$ falls in the category of the optimal solution; in this paper, through the selection of the appropriate $b^{*}, \mathrm{y}_{\mathrm{r}} \mathrm{f}\left(\mathrm{x}_{\mathrm{r}}\right)$ $=1$ is met. In addition, for any $\mathrm{i}$ in it, $\mathrm{C}>\mathrm{a}_{\mathrm{r}}^{*}>0$ is met, and there is correlation between the slack variable definition and the geometric interval as the following $\gamma=\sum_{\mathrm{r}, \mathrm{f} \in \mathrm{I}^{\prime}} \mathrm{y}_{\mathrm{r}} \mathrm{y}_{\mathrm{f}} \mathrm{a}_{\mathrm{r}}{ }^{*} \mathrm{a}_{\mathrm{f}}^{*} \mathrm{~K}\left(\mathrm{x}_{\mathrm{r}^{\prime}} \mathrm{x}_{\mathrm{f}}\right)^{-1 / 2}$

\section{ANALYSIS OF THE COMMUNITY SPORTS MEDICINE HEALTH SERVICE}

\section{Construction of a community sports medicine health system:}

The health of the community sports medicine is dependent mainly on two aspects, viability and development capacity. Therefore, in this paper, the health status of the community sports medicine is measured based on two aspects of viability and development capacity. In accordance with the scientific, comprehensive, data availability and other principles, the community sports medicine health service system is established (Table 1). The indicator system consists of 2 first level indicators, 4 second level indicators and 13 third level indicators and the health of the community sports medicine health system is assessed mainly from 2 aspects of viability and development capacity. Among these, the viability is to measure the current industry status of the community sports medicine health system, including both the scale and growth ability and the medical innovation ability. In the design of the third level indicator, the stock indicator is selected as the half and the incremental indicator is selected as the other half at the beginning of the design. The purpose is to evaluate both the stock and the incremental levels. The development capability is to measure the growth ability 
of the community sports medicine health system in the future, which includes 2 aspects, that is, the investment in the research and development and the input in the technology acquisition and transformation. The third level indicators are mostly input indicators, which are mainly used to evaluate the development potential of the system in the future. In accordance with the availability and timeliness of the data, the time point for the selection of the indicators is selected from 2012 to 2015, and the data are sourced from the China Community Sports Medicine Statistical Yearbook as shown in the following.

\section{Processing of the assessment indicator data on the community sports medicine health:}

The empirical evaluations of the community sports medicine for the scholars both at home and abroad focus on the competitiveness and the industrial upgrading and the methods they have adopted are relatively common, such as the analytic hierarchy process and the fuzzy comprehensive evaluation method. These methods are not only complicated in the calculation process, but also require to be scored by experts to determine the weight of each indicator, hence the objectiveness of the evaluation results is not sufficient. In contrast, the evaluation indicators required by the gait classification algorithm do not include the weight, instead, only the relative importance of the indicators needs to be taken into consideration, which can improve the objectivity of the evaluation results. Another obvious advantage is that there may be collinearity drawback between the indicators. In view of this issue, the gait classification algorithm is selected in this paper to evaluate the health status of the community sports medicine health system in our country. Due to the situation that various indicators in the indicator evaluation system are not consistent in the dimension, in accordance with the requirements for the evaluation of the gait classification algorithm, it is first required to convert the raw data of each control variable into those between the interval $[0,1]$. The following equation is used to carry out the dimensionless data. $Y_{\mathrm{ij}}=\mathrm{X}_{\mathrm{ij}}$-min $\mathrm{X}_{\mathrm{ij} / \max } \mathrm{X}_{\mathrm{ij}-} \min \mathrm{X}_{\mathrm{ij}}$ (in which: $i=1 ; j=1,2 \ldots, 13)$. Hence, the standard values of the viability and the development capacity indicators of the community sports medicine health system can be calculated accordingly, as shown in Tables 2 and 3 .

In accordance with the basic principle of the gait classification algorithm and the mutation model, a comprehensive evaluation of community sports medical health from 2012 to 2015 is carried out. The year 2012 is taken as an example. The evaluation process and the

\section{TABLE 1: COMMUNITY SPORTS MEDICINE HEALTH SERVICE SYSTEM}

\begin{tabular}{lcc}
\hline Target layer & $\begin{array}{c}\text { First level } \\
\text { indicator }\end{array}$ & $\begin{array}{c}\text { Second level } \\
\text { indicator }\end{array}$ \\
& \\
& $\begin{array}{c}\text { Scale and growth } \\
\text { capacity }\left(C_{1}\right)\end{array}$
\end{tabular}
capacity $\left(C_{1}\right)$

Viability $\left(B_{1}\right)$

High technology industry innovation ecosystem health evaluation (A)

$$
\begin{gathered}
\text { Technological } \\
\text { innovation and } \\
\text { output capacity }\left(C_{2}\right)
\end{gathered}
$$

Development capacity $\left(B_{2}\right)$
Investment in research and development $\left(C_{3}\right)$

Technology acquisition and transformation input $\left(C_{4}\right)$
Third level indicator

High technology industry main business income $\left(D_{1}\right)$

High technology industry main business income growth rate $\left(D_{2}\right)$

Number of enterprises in the high technology industry $\left(D_{3}\right)$

Growth rate in the number of enterprises in the high technology industry $\left(D_{4}\right)$

Sales revenue growth of new products in the high technology industry $\left(D_{5}\right)$

Sales revenue growth rate of new products in the high technology industry $\left(D_{6}\right)$

Number of effective patents for invention in the high technology industry $\left(D_{7}\right)$

Growth rate in the number of effective patents for invention in the high technology industry $\left(D_{8}\right)$

R\&D expenditure in the high technology industry $\left(D_{9}\right)$

R\&D personnel full-time equivalent in the high technology industry

$$
\left(D_{10}\right)
$$

New product development funding in the high technology industry $\left(D_{11}\right)$

Technical transformation expenditure of (large and medium-sized) enterprises in the high technology industrial $\left(D_{12}\right)$

Technology introduction and digestion expenditure of (large and medium-sized) enterprises in the high technology industrial $\left(D_{13}\right)$ 
results are shown in Tables 4 and 5. In accordance with the same method and steps, the scores for the 4 second level indicators of the community sports medical health system in other years can be obtained, and the scores of each indicator are ranked (Table 6). The normalized equation of each model is used to synthesize the scores of the indicators at the three levels from bottom up step by step, thus the scores and rankings of 2 first level indicators, viability and development capacity, can be obtained accordingly. In this way, the total scores, specific scores and rankings of the community sports medical health in each year can be obtained (Table 7).

Comparative analysis and conclusions of the community sports medicine health:

In accordance with the results of the ranking of the health status of community sports medicine system in China from 2012 to 2015, combined with the actual situation of the community sports medicine health system in our country, the longitudinal analysis of

TABLE 2: COMMUNITY SPORTS MEDICINE HEALTH SYSTEM VIABILITY STANDARDIZATION DATA IN CHINA FROM 2012 TO 2015

\begin{tabular}{lcccccccc}
\hline Year & \multicolumn{7}{c}{ Third level indicator } \\
\cline { 2 - 9 } 2012 & 0.003301941 & 0.000000001 & 0.000795298 & 0.000000000 & 1.000000000 & 0.000000000 & 0.003159706 & 0.000000010 \\
2013 & 0.002050850 & 0.000000001 & 0.000475277 & 0.000000000 & 1.000000000 & 0.000000002 & 0.002047936 & 0.000000002 \\
2014 & 0.002986651 & 0.000000001 & 0.000655142 & 0.000000000 & 1.000000000 & 0.000000002 & 0.003468747 & 0.000000006 \\
2015 & 0.002364608 & 0.000000001 & 0.000500580 & 0.000000000 & 1.000000000 & 0.000000002 & 0.003374173 & 0.000000005 \\
\hline
\end{tabular}

TABLE 3: COMMUNITY SPORTS MEDICINE HEALTH SYSTEM DEVELOPMENT CAPACITY STANDARDIZATION DATA IN CHINA FROM 2012 TO 2015

\begin{tabular}{lccccc}
\hline Year & \multicolumn{5}{c}{ Third level indicator } \\
\cline { 2 - 6 } 2012 & D9 & D10 & D11 & D12 & D13 \\
2013 & 0.000048144 & 0.000001694 & 0.000058990 & 0.103037389 & 0.026672525 \\
2014 & 0.000030649 & 0.000000986 & 0.000036571 & 0.064879733 & 0.011702798 \\
2015 & 0.000045073 & 0.000001343 & 0.000055118 & 0.074224267 & 0.016793315 \\
\hline
\end{tabular}

TABLE 4: COMMUNITY SPORTS MEDICINE HEALTH ASSESSMENT PROCESS IN 2012

\begin{tabular}{|c|c|c|c|c|}
\hline Type of the system & \multicolumn{4}{|c|}{ Value taken for the variable } \\
\hline \multirow{2}{*}{ Butterfly catastrophe model } & $X_{D 1}$ & $\mathrm{X}_{\mathrm{D} 2}$ & $\mathrm{X}_{\mathrm{D} 3}$ & $X_{D 4}$ \\
\hline & 0.003301941 & 0.000000001 & 0.000795298 & 0.000000000 \\
\hline \multirow{2}{*}{ Butterfly catastrophe model } & $\mathrm{X}_{\mathrm{D} 5}$ & $\mathrm{X}_{\mathrm{D} 6}$ & $\mathrm{X}_{\mathrm{D} 7}$ & $\mathrm{X}_{\mathrm{D} 8}$ \\
\hline & 1.000000000 & 0.000000000 & 0.003159706 & 0.000000010 \\
\hline \multirow{2}{*}{ Swallowtail catastrophe model } & $X_{D g}$ & $X_{D 10}$ & $\mathrm{X}_{\mathrm{D} 11}$ & \\
\hline & 0.000048144 & 0.000001694 & 0.000058990 & \\
\hline \multirow{2}{*}{ Cusp catastrophe model } & $\mathrm{X}_{\mathrm{D} 12}$ & $\mathrm{X}_{\mathrm{D} 13}$ & & \\
\hline & 0.103037389 & 0.026672525 & & \\
\hline \multirow{2}{*}{ Cusp catastrophe model } & $X_{c 1}$ & $X_{c 2}$ & & \\
\hline & 0.237912146 & 0.071927850 & & \\
\hline \multirow{2}{*}{ Cusp catastrophe model } & $\mathrm{X}_{\mathrm{c} 3}$ & $\mathrm{X}_{\mathrm{c} 4}$ & & \\
\hline & 0.188411903 & 0.684698129 & & \\
\hline \multirow{2}{*}{ Cusp catastrophe model } & $\mathrm{X}_{\mathrm{B} 1}$ & $X_{B 2}$ & & \\
\hline & 0.393598778 & 0.434064400 & & \\
\hline
\end{tabular}

TABLE 5: RESULTS OF COMMUNITY SPORTS MEDICINE HEALTH ASSESSMENT IN 2012

\begin{tabular}{llllllll}
\hline Item & $\mathrm{C}_{1}$ & $\mathrm{C}_{2}$ & $\mathrm{C}_{3}$ & $\mathrm{C}_{4}$ & $\mathrm{~B}_{1}$ & $\mathrm{~B}_{2}$ & $A$ \\
\hline
\end{tabular}

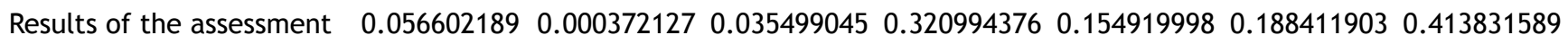


the health status is carried out. The purpose is to improve the understanding of the community sports medicine health system in China. It can be seen from Tables 6 and 7 that in 2012, the scores for the 3 second level indicators, scale growth ability, investment in research and development and input in the technology acquisition and transformation of the community sports medicine health system in China, ranked first; while the medical innovation ability indicator ranked last, hence the development capacity indicator ranked first and the viability indicator ranked fourth, eventually leading to the total score of the healthiness ranks in the third place. Hence, it can be seen that within a certain range, the indicator of the medical innovation ability has a relatively great impact on the viability of the community sports medicine health system, and thus has a relatively great impact on the total score of the healthiness. This inference is also reflected in the data in 2013. In the same year, the scores for all the three indicators, that is, the scale growth ability, the investment in research and development and the input in technology acquisition and transformation of the community sports medicine health system, rank fourth; while the indicator of medical innovation ability ranks in second place; the development capacity indicator ranks at the bottom, but the viability indicator rises to the second place, which eventually leads to the rise of its ranking in the overall health score to the third place. In addition, as shown in Table 6, in 2012 the input in the technology acquisition and transformation of the community sports medicine health system in China was at the highest level in history. Since then, it has been presenting a downward trend and fluctuating significantly. However, the medical innovation ability indicator is at the lowest level in history and has remained basically stable since then; the fluctuations in the scale growth capacity and the investment in research and development are relatively small as well. It can be inferred that the degree of fluctuation in the input in the technology acquisition and transformation can have a relatively great impact on the health status of the community sports medicine health system.

As shown in Table 7, in 2014, the total score of the community sports medicine health is the highest, and its health service ability indicators are among the highest in the ranking. Compared to that in 2013, both indicators showed an upward trend, which suggested that the community sports medicine health system at this time is in a relatively healthy state compared to recent years; while in 2015, the total score of the community sports medicine health ranked in the second place. After analysis, it is found that the reason is that the health service capacity indicator is in a downward trend, which resulted in a slight decrease in the total health score. For the year 2012, although the development indicator of the community sports medicine health system is at the highest point in the past 4 years, it may be because its viability is at the lowest point in the past 4 years and the side effects are relatively large, thus its total score of the healthiness is reduced. As the viability indicator and the development indicator of the community sports medicine health system in 2013 are at the middle and lower level in 4 years, the total score of the healthiness in this year is at the lowest value in the recent years. In general, it can be seen that when the viability and development capacity are increasing in the positive direction, the total score of the health status of the

TABLE 6: SCORES AND RANKINGS OF THE SECOND LEVEL INDICATORS OF THE COMMUNITY SPORTS MEDICINE HEALTH SYSTEM IN CHINA FROM 2012 TO 2015

\begin{tabular}{ccccccccc}
\hline \multirow{2}{*}{ Year } & \multicolumn{2}{l}{ Scale and growth capacity } & $\begin{array}{c}\text { Technological innovation } \\
\text { and output capacity }\end{array}$ & \multicolumn{2}{c}{$\begin{array}{c}\text { Investment in research } \\
\text { and dvelopment }\end{array}$} & \multicolumn{2}{c}{$\begin{array}{c}\text { Technology acquisition } \\
\text { and transformation input }\end{array}$} \\
\cline { 2 - 9 } & Score/point & $\begin{array}{c}\text { Ranking/ } \\
\text { rank }\end{array}$ & Score/point & $\begin{array}{c}\text { Ranking/ } \\
\text { rank }\end{array}$ & Score/point & $\begin{array}{c}\text { Ranking/ } \\
\text { rank }\end{array}$ & Score/point & $\begin{array}{c}\text { Ranking/ } \\
\text { rank }\end{array}$ \\
2012 & 0.056602189 & 1 & 0.000372127 & 4 & 0.035499045 & 1 & 0.320994376 & 1 \\
2013 & 0.048462356 & 4 & 0.001318268 & 2 & 0.031085096 & 4 & 0.227036963 & 4 \\
2014 & 0.053937303 & 2 & 0.001318272 & 1 & 0.034636499 & 2 & 0.256081856 & 2 \\
2015 & 0.049767740 & 3 & 0.001215116 & 3 & 0.032439602 & 3 & 0.238098787 & 3 \\
\hline
\end{tabular}

TABLE 7: SCORES AND RANKINGS OF THE FIRST LEVEL INDICATORS OF THE COMMUNITY SPORTS MEDICINE HEALTH SYSTEM IN CHINA FROM 2012 TO 2015

\begin{tabular}{ccccccc}
\hline \multirow{2}{*}{ Year } & \multicolumn{2}{c}{ Viability } & \multicolumn{2}{c}{ Development capacity } & \multicolumn{2}{c}{ Healthiness } \\
\cline { 2 - 7 } 2012 & Score/point & Ranking/rank & Score/point & Ranking/rank & Score/point & Ranking/rank \\
2013 & 0.154919998 & 4 & 0.188411903 & 1 & 0.413831589 & 3 \\
2014 & 0.164894907 & 3 & 0.176309661 & 4 & 0.412982489 \\
2015 & 0.170946150 & 1 & 0.186108837 & 2 & 0.422429840 & 1 \\
\hline
\end{tabular}


community sports medicine system in our country is on the rise; and the higher the health score is, the healthier the system is. Certainly, it can also be seen from Table 7 that the health status scores of the community sports medicine system in our country have been below 0.5 in the past 4 years, which indicates that the health status of the community sports medicine system in our country is insufficient at present, and further cultivation and development are required.

In summary, two conclusions can be drawn, firstly, the health level of the community sports medicine health system in China is relatively general and needs to be further improved; secondly, within a certain range, the medical innovation ability and the input in the technology acquisition and transformation have relatively great impact on the health status of the community sports medicine health system.

\section{Empirical analysis of the community sports medicine health service system:}

On the basis of the above empirical analysis on the community sports medicine health system, combined with the achievements of the previous scholars on the community sports medicine health system, countermeasures and suggestions are put forward in this paper from three aspects as the following: improving the medical innovation ability, increasing the input in the technology acquisition and transformation and building an open good community sports medicine health service system.

\section{Improve the medical innovation ability:}

In order to develop the community sports medicine health system, it is necessary to first improve the medical innovation ability. While increasing the support for the basic research and applied research, it is necessary to focus on the macro policy support that guides the direction of the industrial development at the same time, as well as the micro policy of the investment mechanisms, as well as the innovation and entrepreneurship incentive mechanisms, so as to create a good policy environment for the improvement of the medical innovation and output capacity. Secondly, it is necessary to optimize the organizational structure of the industrial innovation, enrich the main types of the industrial innovation, change the innovation situation where only single subjects of the scientific research institutions and community sports medicine are present, and establish a five-in-one joint innovation system of "Government, industry, research and application", so as to provide support for the improvement of the medical innovation ability. Finally, it is necessary to give full play to the coordination role of the intermediary organizations, strengthen the role of the market entities such as the headhunting companies and risk assessment agencies in the community sports medicine health system, increase the vitality of the community sports medicine innovation subjects, reduce the risk of the community sports medicine innovation, and provide guarantee for the improvement of the medical innovation ability.

\section{Increase the input in the technology acquisition and transformation:}

The development of the community sports medicine health system requires continuous innovation. In addition, it is inseparable from large amount and continuous technical investment. On the one hand, it is necessary to vigorously strengthen the technology acquisition, spread the scope of the community sports medicine health system to overseas, actively connect with the community sports medicine and key laboratories abroad, and obtain the latest products through the technical cooperation, joint holding, joint ventures and other measures, so as to understand the latest direction of the technological innovation. In addition, active efforts should be made to form technical alliances with overseas scientific research institutions, so as to disperse the huge risks brought about by the technological innovation. On the other hand, it is necessary to increase the input in the technological transformation and vigorously improve the self-transformation capacity of technology, which not only requires to associate with domestic colleges, universities, research institutes and community sports medicine to thoroughly digest the new foreign technologies, but also requires to carry out iteration on the own core technologies, so as to improve the capacity of the community sports medicine health system for the transformation and upgrading of the new technologies in an effective manner from both internal and external perspectives.

The subjects of the community sports medicine innovation must achieve symbiosis through mutual cooperation to form an open and group type innovation model. The closed and single development model can no longer match with the community sports medicine health system that is characterized by symbiosis and openness. Only by creating an open and inclusive community sports medicine health service system can the innovation subjects carry out exchange through the material flow, knowledge flow and information flow with the external environment, so as to acquire the 
materials, knowledge and technologies that are required in the system development process. In addition, it is necessary to expand the openness, lower the barriers to entry for the subjects of the community sports medicine and enhance the capacity to absorb new members, so as to continuously inject fresh blood for the development of the community sports medicine health system. The construction of an open community sports medicine health service system includes not only the opening up of the industrial development subjects to the external environment, but also the opening up between the industrial subjects. Exchange and cooperation shall be carried out to achieve the advantage complementation, conduct proactive learning and communication and enhance the diffusion effect of the knowledge chain, so as to form a favorite atmosphere for innovation.

\section{ACKNOWLEDGEMENTS}

This study was supported by Science and Technology Department of Sichuan Province (Key Program), No. 2017SZ0018.

\section{REFERENCES}

1. Donath L, Roth RC, Hürlimann, Zahner L, Faude O. Pilates vs. balance training in health community-dwelling seniors: a 3-arm, randomized controlled trial. Int J Sports Med 2016;37(03):202-10.

2. Joy E, Blair SN, Mcbride P, Sallis R. Physical activity counselling in sports medicine: a call to action. Br J Sports Med 2013;47(1):49-53.

3. Finch CF, Diamantopoulou K, Twomey DM, Doyle TLA, Lloyd DG, Young W. The reach and adoption of a coach-led exercise training programme in community football. $\mathrm{Br} \mathrm{J}$ Sports Med 2014;48(8):718-23.

4. Chwastiak L, Cruza-Guet MC, Carroll-Scott A, Sernyak M, Ickovics J. Preventive counseling for chronic disease: missed opportunities in a community mental health center. Psychosomatics 2013;54(4):328-35.

5. Khan KM. Bjsm: serving national sports medicine and sports physiotherapy societies and major international sporting organisations. Br J Sports Med 2014;48(12):939-40.

6. Kümmel J, Kramer A, Giboin LS, Gruber M. Specificity of balance training in healthy individuals: a systematic review and meta-analysis. Sports Med 2016;46(9):1261-71.

7. Ekstrand J. Keeping your top players on the pitch: the key to football medicine at a professional level. Br J Sports Med 2013;47(12):723-4.

8. Evans MF. ' 23 and $1 / 2$ h' goes viral: top 10 learnings about making a health message that people give to one another. $\mathrm{Br} \mathrm{J}$ Sports Med 2012;46(46):461-2.

9. Hollis SJ, Stevenson MR, Mcintosh AS, Shores EA, Finch CF. Compliance with return-to-play regulations following concussion in australian schoolboy and community rugby union players. Br J Sports Med 2012; 46(10):735-40.

10. Tapkan P, Özbakır L, Kulluk S, Baykasoğlu A. A cost-sensitive classification algorithm: bee-miner. KNOWL-BASED SYST 2016;95:99-113.

11. Wang L, Ji HB, Jin Y. Fuzzy passive-aggressive classification: a robust and efficient algorithm for online classification problems. Inf Sci 2013;220:46-63.

12. Muhlhaus MS, Oner M, Dobre OA, Jondral FK. A low complexity modulation classification algorithm for mimo systems. IEEE COMMUN LETT 2013;17(10):1881-4.

13. Phillips RD, Watson LT, Wynne RH. An smp soft classification algorithm for remote sensing. COMPUT GEOSCI 2014;68:7380.

14. Yahyaoui H, Al-Mutairi, A. A feature-based trust sequence classification algorithm. Inf Sci 2016; 328:455-84.

15. Yin C, Feng L, Ma, L. An improved hoeffding-id data-stream classification algorithm. J Supercomput 2016;72(7):2670-81.

This is an open access article distributed under the terms of the Creative Commons Attribution-NonCommercial-ShareAlike 3.0 License, which allows others to remix, tweak, and build upon the work non-commercially, as long as the author is credited and the new creations are licensed under the identical terms

This article was originally published in a special issue: Special issue on "Animal Models \& Experimental Medicine"

Indian J Pharm Sci 2020:82(1)spl issue4;10-18 